\title{
Application of the Revision Theory to Adaptation in Case-Based Reasoning: the Conservative Adaptation
}

\author{
Jean Lieber \\ LORIA (UMR 7503 CNRS-INRIA-Nancy Universities), \\ BP 239, 54506 Vandœuvre-lès-Nancy, FRANCE \\ lieber@loria.fr
}

\begin{abstract}
Case-based reasoning aims at solving a problem by the adaptation of the solution of an already solved problem that has been retrieved in a case base. This paper defines an approach to adaptation called conservative adaptation; it consists in keeping as much as possible from the solution to be adapted, while being consistent with the domain knowledge. This idea can be related to the theory of revision: the revision of an old knowledge base by a new one consists in making a minimal change on the former, while being consistent with the latter. This leads to a formalization of conservative adaptation based on a revision operator in propositional logic. Then, this theory of conservative adaptation is confronted to an application of case-based decision support to oncology: a problem of this application is the description of a patient ill with breast cancer, and a solution, the therapeutic recommendation for this patient. Examples of adaptations that have actually been performed by experts and that can be captured by conservative adaptation are presented. These examples show a way of adapting contraindicated treatment recommendations and treatment recommendations that cannot be applied.
\end{abstract}

Keywords: case-based reasoning, knowledge-intensive case-based reasoning, adaptation, conservative adaptation, theory of revision, logical representation of cases, application to oncology

\section{Introduction}

Case-based reasoning (CBR [1]) aims at solving a new problem thanks to a set of already solved problems. The new problem is called the target problem, denoted by tgt in this paper, and the already solved problems are the source problems, denoted by srce. A case is the representation of a problem-solving episode, that is, at least a problem $\mathrm{pb}$ and a solution $\mathrm{Sol}(\mathrm{pb})$ of $\mathrm{pb}$. Hence a case is denoted by a pair $(\mathrm{pb}, \mathrm{Sol}(\mathrm{pb}))$. A source problem srce is a problem that has already been solved in a solution Sol(srce). The pair (srce, Sol(srce)) is a source case and the set of source cases is the case base. A classical decomposition of the CBR inference points out three steps: retrieval, adaptation and memorization. Retrieval selects a source case ( $\operatorname{srce}, \operatorname{Sol}(\operatorname{srce}))$ that is judged similar to tgt, according to some similarity criterion. Adaptation aims at solving tgt thanks to the retrieved case (srce, Sol(srce)). Thus, a successful adaptation provides 
a solution Sol(tgt) to tgt, in general by modification of Sol(srce). Finally, memorization evaluates the utility of storing the new case (tgt, Sol(tgt)) in the case base and stores it when it is useful. Knowledge-intensive approaches of CBR are such that the domain knowledge plays a key role (and not only the case base) [2]. This holds for the conservative adaptation as it is shown hereafter.

\subsection{CBR and Adaptation}

In general, it is considered that the CBR inference is based on the following principle:

Similar problems have similar solutions.

(CBR principle)

This principle has been formalized in [3] by

$$
\mathcal{T}(\text { Sol }(\text { srce }), \text { Sol }(\text { tgt })) \geq \mathcal{S}(\text { srce, tgt })
$$

(translated with our notations) where $\mathcal{S}$ and $\mathcal{T}$ are similarity measures respectively between problems and solutions: the solution Sol(tgt) is constrained to be similar to Sol(srce). There are multiple ways of specifying the adaptation step in accordance to the CBR principle, starting from the so-called null adaptation:

$$
\operatorname{Sol}(\text { tgt }):=\operatorname{Sol}(\text { srce) } \quad \text { (null adaptation) }
$$

Null adaptation is justified in [1] by the fact that "people often do very little adaptation". One limit of null adaptation is that the fact "Sol(srce) solves tgt" may contradict some domain knowledge. In this case, a strategy for adaptation is the following:

Sol(tgt) is obtained by keeping from Sol(srce) as much as possible features

while keeping the available knowledge consistent. (conservative adaptation)

Conservative adaptation aims at following the CBR principle in the sense that it tends to make the similarity $\mathcal{T}$ (Sol(srce), Sol(tgt)) maximal.

\subsection{Overview of the Paper}

In section 2, the principle of conservative adaptation is presented with more details. It relates this kind of adaptation with the theory of revision: both of them are based on minimal change. Section 3 presents the basic principles of the theory of revision. This theory consists of a set of axioms that a revision operator has to satisfy. Section 4 provides a formalization of conservative adaptation based on a given revision operator. This work is motivated by an application in oncology: the KASIMIR system, in which a problem represents a class of patients and a solution represents a treatment proposal for these patients. From our study of adaptations actually performed by experts in oncology, several adaptation patterns have emerged [4]. Several of these patterns can be implemented thanks to conservative adaptation; this is what is illustrated in section 5 . Section 6 discusses this work. Finally, section 7 draws some conclusions and points out new directions of work following this study. 


\section{Principle of Conservative Adaptation}

Let us consider the following example of conservative adaptation:

Example 1 Léon is about to invite Thècle and wants to prepare her an appropriate meal. His target problem can be specified by the characteristics of Thècle about food. Let us assume that Thècle is vegetarian (denoted by the propositional variable $v$ ) and that she has other characteristics (denoted by o) not detailed in this example: tgt $=$ $v \wedge$ o. From his experience as a host, Léon remembers that he had invited Simone some times ago and he thinks that Simone is very similar to Thècle according to food behavior, except that she is not a vegetarian: srce $=\neg v \wedge o$. He had proposed to Simone a meal with salad (s), beef (b) and a dessert (d), and she was satisfied by the two formers but has not eaten the dessert, thus Léon has retained the case (srce, Sol(srce)) with Sol $($ srce $)=s \wedge b \wedge \neg d$. Besides that, Léon has some general knowledge about food: he knows that beef is meat, that meat and tofu are protein foods, and that vegetarians do not eat meat. Thus, his domain knowledge is

$$
D K_{\text {Léon }}=b \Rightarrow m \wedge m \Rightarrow p \wedge t \Rightarrow p \wedge v \Rightarrow \neg m
$$

where $b, m, t$ and $p$ are the propositional variables for "some beef/meat/tofu/protein food is appreciated by the current guest". According to conservative adaptation, what meal should be proposed to Thècle? Sol(srce) itself is not a satisfactory solution of tgt: Sol(srce) $\wedge \operatorname{tgt} \wedge D K_{\text {Léon }}$ is unsatisfiable. However, the features $s$ and $\neg d$ can be kept in Sol(srce) to solve tgt. Moreover, what conducts to a contradiction is the fact that there is a meat, not in the fact that it is a protein food. Therefore, a solution of tgt according to conservative adaptation could be $s \wedge p \wedge \neg d$. Another one could be to replace beef by tofu: $s \wedge t \wedge \neg d$.

As this example illustrates, the adaptation process consists of a shifting from the source context to the target context. If this process is conservative, then this shifting has to operate a minimal change and, in the same time, must be consistent with the definition of the target problem. Both contexts are interpreted in the framework of the "permanent knowledge", i.e., the knowledge of the CBR system, i.e., the domain knowledge. Therefore, conservative adaptation is based on three kinds of knowledge:

$\left(\mathrm{KB}_{1}\right)$ The old knowledge that can be altered (but must be altered minimally): the knowledge related to the context of the source problem and its solution;

$\left(\mathrm{KB}_{2}\right)$ The new knowledge, that must not be altered during the process: the knowledge related to the context of the target problem;

(DK) The knowledge that is permanent (true in any context): the domain knowledge (i.e., the general knowledge of the domain of the CBR system under consideration, e.g., the ontology giving the vocabulary with which the cases are expressed).

The question that is raised is "What is the minimal change on the knowledge base $\mathrm{KB}_{1}$ that must be done to be consistent with knowledge base $\mathrm{KB}_{2}$ ?" When $\mathrm{KB}_{1}$ and $\mathrm{KB}_{2}$ do not contradict, there is no reason to change $\mathrm{KB}_{1}$ and thus, a conservative adaptation process entails $\mathrm{KB}_{1}$, which amounts to a null adaptation. 
This principle of minimal change of knowledge can be found in the theory of $r e$ vision: given two knowledge bases $\psi$ and $\mu$, the revision of $\psi$ by $\mu$ is a knowledge base $\psi \circ \mu$ that entails $\mu$ and makes the minimal change on $\psi$ to make this revision consistent [5].

Both $\mathrm{KB}_{1}$ and $\mathrm{KB}_{2}$ must be interpreted in consistency with the domain knowledge DK. Thus, conservative adaptation consists, given a revision operator $\circ$, in computing $\left(\mathrm{DK} \wedge \mathrm{KB}_{1}\right) \circ\left(\mathrm{DK} \wedge \mathrm{KB}_{2}\right)$ and to infer from this new knowledge base the pieces of information that are relevant to Sol(tgt).

So, before formalizing conservative adaptation, it is necessary to introduce the notion of revision operator.

\section{Revision of a Knowledge Base}

Revision of a knowledge base has been formalized independently from a particular logic in the so-called AGM theory (called after the initials of the [5]'s authors). This theory has been applied, in particular, to propositional logic by [6] and it is this work that is presented here, since the current paper concentrates on this formalism.

\subsection{Preliminaries}

The propositional formulas are assumed to be built on $\mathcal{V}$, a finite set of propositional variables. An interpretation $\mathcal{I}$ is a function from $\mathcal{V}$ to the pair $\{$ true, false $\}$. If $a \in \mathcal{V}$, $\mathcal{I}(a)$ is also denoted by $a^{\mathcal{I}} . \mathcal{I}$ is extended on the set of formulas in the usual way $\left((f \wedge g)^{\mathcal{I}}=\right.$ true iff $f^{\mathcal{I}}=$ true and $g^{\mathcal{I}}=$ true, etc. $)$. A model of a formula $f$ is an interpretation $\mathcal{I}$ such that $f^{\mathcal{I}}=\operatorname{true}$. $\operatorname{Mod}(f)$ denotes the set of models of $f . f$ is satisfiable means that $\operatorname{Mod}(f) \neq \emptyset$. $f$ entails $g$ (resp., $f$ is equivalent to $g$ ), denoted by $f \vDash g$ (resp., $f \equiv g)$, if $\operatorname{Mod}(f) \subseteq \operatorname{Mod}(g)(\operatorname{resp}$., $\operatorname{Mod}(f)=\operatorname{Mod}(g))$, for two formulas $f$ and $g$. Finally, $g \vDash_{f} h$ (resp., $g \equiv_{f} h$ ) means that $g$ entails $h$ (resp., $g$ is equivalent to $h$ ) under $f: f \wedge g \vDash h$ (resp., $f \wedge g \equiv f \wedge h$ ).

\subsection{Katsuno and Mendelzon's Axioms}

Let $\circ$ be a revision operator. $\psi \circ \mu$ is a formula expressing the revision of $\psi$ by $\mu$, according to the operator $\circ: \psi$ is the "old" knowledge base (that has to be revised), $\mu$ is the "new" knowledge base (that contains knowledge revising the old one). The axioms that a revision operator on propositional logic has to satisfy are:

(R1) $\psi \circ \mu \vDash \mu$ (the revision operator has to retain all the knowledge of the new knowledge base $\mu$ );

(R2) If $\psi \wedge \mu$ is satisfiable, then $\psi \circ \mu \equiv \psi \wedge \mu$ (if the new knowledge base does not contradict the old one, then every piece of knowledge of the two bases has to be kept);

(R3) If $\mu$ is satisfiable then $\psi \circ \mu$ is also satisfiable (o does not lead to an unsatisfiable knowledge base, unless the new knowledge is itself unsatisfiable);

(R4) If $\psi \equiv \psi^{\prime}$ and $\mu \equiv \mu^{\prime}$ then $\psi \circ \mu \equiv \psi^{\prime} \circ \mu^{\prime}$ (the revision operator follows the principle of irrelevance of syntax); 
(R5) $(\psi \circ \mu) \wedge \phi \vDash \psi \circ(\mu \wedge \phi)$;

(R6) If $(\psi \circ \mu) \wedge \phi$ is satisfiable then $\psi \circ(\mu \wedge \phi) \vDash(\psi \circ \mu) \wedge \phi$.

for $\psi, \psi^{\prime}, \mu, \mu^{\prime}$, and $\phi$, five propositional formulas. (R5) and (R6) are less obvious to understand then (R1) to (R4) and are explained in [6]. They are linked with the idea that a revision operator is supposed to perform a minimal change: $\psi \circ \mu$ keeps "as much as possible" from $\psi$ while being consistent with $\mu$.

\subsection{Distance-based Revision Operators and Dalal's Revision Operator}

In [6], a characterization and a survey of revision operators in propositional logic is proposed. This paper highlights a class of revision operators based on distances between interpretations. Let dist be such a distance. For $M_{1}$ and $M_{2}$ two sets of interpretations and $\mathcal{J}$ an interpretation,

$$
\begin{aligned}
\text { let } \operatorname{dist}\left(M_{1}, \mathcal{J}\right) & =\min \left\{\operatorname{dist}(\mathcal{I}, \mathcal{J}) \mid \mathcal{I} \in M_{1}\right\} \\
\text { and } \operatorname{dist}\left(M_{1}, M_{2}\right) & =\min \left\{\operatorname{dist}\left(M_{1}, \mathcal{J}\right) \mid \mathcal{J} \in M_{2}\right\} \\
& =\min \left\{\operatorname{dist}(\mathcal{I}, \mathcal{J}) \mid \mathcal{I} \in M_{1} \text { and } \mathcal{J} \in M_{2}\right\}
\end{aligned}
$$

Now let $\psi$ and $\mu$ be two formulas and $\Delta=\operatorname{dist}(\operatorname{Mod}(\psi), \operatorname{Mod}(\mu))$. Then, the revision operator $\circ_{\text {dist }}$ based on dist is defined by

$$
\operatorname{Mod}\left(\psi \circ_{\text {dist }} \mu\right)=\{\mathcal{J} \mid \mathcal{J} \in \operatorname{Mod}(\mu) \text { and } \operatorname{dist}(\operatorname{Mod}(\psi), \mathcal{J})=\Delta\}
$$

This equation defines $\psi \circ_{\text {dist }} \mu$ up to the equivalence between formulas (since we adhere to the principle of irrelevance of syntax, this is sufficient). The proof that axioms (R1) to (R6) hold for $\circ_{\text {dist }}$ is a rather straightforward application of the definitions above. Note, in particular, that (R2) can be proven thanks to the equivalence $\operatorname{dist}(\mathcal{I}, \mathcal{J})=0$ iff $\mathcal{I}=\mathcal{J}$, for two interpretations $\mathcal{I}$ and $\mathcal{J}$.

The intuition of minimal change from $\psi$ to $\psi \circ_{\text {dist }} \mu$ is related to the distance dist between interpretations: $\psi \circ_{\text {dist }} \mu$ is the knowledge base whose interpretations are the interpretations of $\mu$ that are the closest ones to those of $\psi$, according to dist.

The Dalal's revision operator $\circ_{D}[7]$ is such a revision operator: it corresponds to the Hamming distance between interpretations defined by: $\operatorname{dist}(\mathcal{I}, \mathcal{J})$ is the number of propositional variable $a \in \mathcal{V}$ such that $a^{\mathcal{I}} \neq a^{\mathcal{J}}$. It is this operator that has been chosen for the examples of this paper.

\section{Formalization of Conservative Adaptation}

This section presents a formalization of conservative adaptation based on a revision operator in propositional logic, an example using Dalal's revision operator, and a discussion on the meaning of Katsuno and Mendelzon's axioms for conservative adaptation. 


\subsection{Conservative Adaptation Process based on a Revision Operator}

It is assumed that all the knowledge entities of the CBR system under consideration (problem, solution, domain knowledge) are represented in the formalism of propositional logic. The natural language assertion "pb is the current problem" is translated simply in $\mathrm{pb}$. From this and the informal definition of conservative adaptation presented in section 1.1, it comes that, in order to solve tgt by conservative adaptation of (srce, Sol(srce)), the following knowledge bases are defined:

$$
\mathrm{KB}_{1}=\operatorname{srce} \wedge \operatorname{Sol}(\text { srce }) \quad \mathrm{KB}_{2}=\operatorname{tgt}
$$

Let $\circ$ be a revision operator. The $\circ$-conservative adaptation consists in computing TSKCA $=\left(\mathrm{DK} \wedge \mathrm{KB}_{1}\right) \circ\left(\mathrm{DK} \wedge \mathrm{KB}_{2}\right)$, where $\mathrm{DK}$ denotes the domain knowledge, and, second, entails from TSKCA pieces of information relevant to solve tgt (TSKCA is the target solution knowledge inferred by conservative adaptation).

\subsection{Example}

From this principle, the example 1 (section 2) can be treated as follows. The knowledge bases $\mathrm{DK}, \mathrm{KB}_{1}$, and $\mathrm{KB}_{2}$ are:

$$
\mathrm{DK}=\mathrm{DK}_{\mathrm{Léon}} \quad \mathrm{KB}_{1}=\neg v \wedge o \wedge s \wedge b \wedge \neg d \quad \mathrm{~KB}_{2}=v \wedge o
$$

With $\circ_{\mathrm{D}}$, the Dalal's revision operator on propositional logic (see section 3), it can be proven that

$$
\mathrm{TSKCA}=\left(\mathrm{DK} \wedge \mathrm{KB}_{1}\right){ }^{\circ} \mathrm{D}\left(\mathrm{DK} \wedge \mathrm{KB}_{2}\right) \equiv_{\mathrm{DK}_{\mathrm{L} \text { Ló }}} \underbrace{v \wedge o} \wedge \underbrace{s \wedge \neg b \wedge \neg m \wedge p \wedge \neg d}
$$

(a)

The target problem tgt $=v \wedge o=(\mathrm{a})$ is entailed by TSKCA: this is true for any revision operator. Indeed, from axiom (R1), TSKCA $\vDash \mathrm{DK} \wedge \mathrm{KB}_{2}$, and $\mathrm{DK} \wedge \mathrm{KB}_{2} \vDash$ tgt (since $\mathrm{KB}_{2}=$ tgt).

In the example 1, two plausible solutions were proposed: $\mathrm{Sol}_{1}$ (tgt) $=s \wedge p \wedge \neg d$ and $\mathrm{Sol}_{2}(\mathrm{tgt})=s \wedge t \wedge \neg d$. The former can be entailed from TSKCA: (b) $\vDash \mathrm{Sol}_{1}$ (tgt). But (b) indicates more precisely that some protein food that is not meat $(\neg m \wedge p)$ is appreciated by the guest. This does not involve that the guest appreciates tofu. Now, let $\mathrm{DK}_{\mathrm{Léon}}^{\prime}$ be the knowledge of Léon with the additional knowledge that the only available protein food of Léon that is not meat is tofu: $\mathrm{DK}_{\text {Léon }}^{\prime}=\mathrm{DK}_{\mathrm{Léon}} \wedge(p \Rightarrow m \vee t)$. By substituting $\mathrm{DK}_{\mathrm{Léon}}$ by $\mathrm{DK}_{\mathrm{Léon}}^{\prime}$ it comes:

$\mathrm{TSKCA}^{\prime}=\left(\mathrm{DK}_{\mathrm{Léon}}^{\prime} \wedge \mathrm{KB}_{1}\right) \circ_{\mathrm{D}}\left(\mathrm{DK}_{\mathrm{Léon}^{\prime}}^{\prime} \wedge \mathrm{KB}_{2}\right) \equiv_{\mathrm{DK}_{\mathrm{Leon}}^{\prime}} \underbrace{v \wedge o} \wedge \underbrace{s \wedge \neg b \neg m \wedge t \wedge p \wedge \neg d}$

(a)

and $\left(b^{\prime}\right) \vDash \mathrm{Sol}_{2}$ (tgt) . 


\subsection{Revision Axioms and Conservative Adaptation}

Now, the Katsuno and Mendelzon's axioms (R1) to (R6) can be reconsidered at the light of conservative adaptation.

(R1) applied to conservative adaptation gives TSKCA $\vDash D K \wedge$ tgt. If this assertion were violated, this would mean that there exists a model $\mathcal{I}$ of TSKCA such that $\mathcal{I} \notin$ $\operatorname{Mod}(\mathrm{DK} \wedge \mathrm{tgt})=\operatorname{Mod}(\mathrm{DK}) \cap \operatorname{Mod}(\operatorname{tg} t)$. Therefore $\mathcal{I}$ would contradict

- Either the definition of the target problem (meaning that the conservative adaptation solves another target problem!);

- Or the domain knowledge (that has to be preserved by conservative adaptation).

Thus, using a revision operator that satisfies (R1) prevents from these two kinds of contradiction.

Let us assume that $D K \wedge K B_{1} \wedge K_{2}$ is satisfiable: in other words srce $\wedge$ Sol (srce $) \wedge$ tgt is consistent under the domain knowledge DK. Then, (R2) entails that TSKCA $\equiv$ $\mathrm{DK} \wedge \mathrm{KB}_{1} \wedge \mathrm{KB}_{2}$. Thus, TSKCA $\vDash \operatorname{srce} \wedge \operatorname{Sol}($ srce $) \wedge$ tgt: if tgt is consistent with srce $\wedge$ Sol(srce) in DK, then it can be inferred by conservative adaptation that Sol(srce) solves tgt. This is consistent with the principle of this kind of adaptation: Sol(tgt) is obtained by keeping from $\mathrm{Sol}(\mathrm{srce})$ as much as possible, and if the fact "Sol(srce) solves tgt" does not contradict DK, then conservative adaptation amounts to null adaptation.

(R3) gives: if $D K \wedge K B_{2}$ is satisfiable then TSKCA is satisfiable. The satisfiability of $\mathrm{DK} \wedge \mathrm{KB}_{2}=\mathrm{DK} \wedge$ tgt means that the specification of the target problem does not contradict the domain knowledge. Thus, (R3) involves that whenever the target problem is specified in accordance with the CBR domain knowledge, conservative adaptation provides a satisfiable result.

(R4) simply means that conservative adaptation follows the principle of irrelevance of syntax.

The conjunction of (R5) and (R6) can be reformulated as follows:

- Either $(\psi \circ \mu) \wedge \phi$ is unsatisfiable,

- $\operatorname{Or}(\psi \circ \mu) \wedge \phi \equiv \psi \circ(\mu \wedge \phi)$.

Applied to conservative adaptation, it gives:

- Either TSKCA $\wedge \phi$ is unsatisfiable,

- Or TSKCA $\wedge \phi \equiv\left(\mathrm{DK} \wedge \mathrm{KB}_{1}\right) \circ\left(\mathrm{DK} \wedge \mathrm{KB}_{2} \wedge \phi\right)$.

Let $\phi$ be a formula representing some additional knowledge about the target problem. If $\phi$ is consistent with the result of conservative adaptation (TSKCA is satisfiable) then the conjunction of (R5) and (R6) entails that adding $\phi$ to tgt before the conservative adaptation process or after it gives the same result.

\section{Application: Conservative Adaptation of Breast Cancer Treatments}

The KASIMIR project aims at the management of decision protocols in oncology. Such decision protocols have to be adapted for some medical cases. This section shows some 
examples of adaptations performed by experts (oncologists) and how these examples can be modeled by conservative adaptation.

\subsection{The Kasimir Project}

A huge research effort has been put on oncology during the last decades. As a consequence, the complexity of decision support in oncology has greatly increased. The KASIMIR project aims at the management of decision knowledge in oncology. A big part of this knowledge is constituted by decision protocols. For example, the protocol for breast cancer treatment is a document indicating how a patient suffering from this disease has to be treated. Therefore, this protocol can be seen as a set of rules Pat $\longrightarrow$ Ttt, where Pat denotes a class of patients and Ttt, a treatment for the patients in Pat.

Unfortunately, for about one third of the patients, this protocol cannot be applied, for example because of contraindications (other examples are presented in section 5.3). Indeed, it is practically impossible to list all the specific situations that prevent the application of the protocol: this is an instance of what [8] calls the qualification problem. It has been shown that, in these situations, the oncologists often adapt the protocol for recommending a treatment to these patients (meaning that they reuse the protocol, but not in a straightforward manner). More precisely, given the description of a target patient, tgt, a rule Pat $\longrightarrow$ Ttt is selected in the protocol, such that Pat is similar to tgt, and Ttt is adapted to fit the particularities of tgt. If the rules Pat $\longrightarrow$ Ttt are assimilated to source cases $(\operatorname{srce}, \operatorname{Sol}(\operatorname{srce}))-$ srce $=$ Pat and Sol $($ srce $)=$ Tttthen this process is an instance of $\mathrm{CBR}$, with the particularity that the source cases are generalized cases (as called in [9]), also known as ossified cases (in [1]).

\subsection{The KASIMIR System}

The KASIMIR system aims at assisting physicians in their decision making process. The last version of this system has been implemented as a semantic portal (i.e., a portal of the semantic Web [10]), using as representation language the $\mathrm{W} 3 \mathrm{C}$ recommendation OWL DL that is equivalent to the expressive description logic $\mathcal{S H O I N ( D ) ~ [ 1 1 ] . ~}$

This system performs protocol application: given a protocol written in OWL DL and the description of a patient, it highlights the treatments that the protocol recommends to the patient. It also implements adaptation processes, based on some adaptation knowledge [12]. Current studies aim at acquiring this adaptation knowledge: from experts [4] and semi-automatically [13].

Conservative adaptation appears as a promising research direction for adaptation within the KASIMIR system, as next section shows.

\subsection{Examples}

Two examples corresponding to real situations of decision problems of breast cancer treatment are presented below, followed by an explanation in term of conservative adaptation expressed in propositional logic. The first one deals with the adaptation of a contraindicated treatment. The second one deals with the adaptation of an inapplicable treatment. Other examples of conservative adaptation related to KASIMIR are presented in the research report [14]. 
Example 2 Some hormones of the human body facilitate the development of cells. In particular, oestrogens facilitate the growing of some breast cells, including some cancerous breast cells. A hormonotherapy is a long term treatment that aims at inhibiting the development of hormons (or their actions) to lower the chance of having a new tumor developed after the other types of treatment (surgery, chemotherapy and radiotherapy) have been applied. Tamoxifen is a hormonotherapy drug that prevents from the action of oestrogen on breast cells. Unfortunately, tamoxifen is contraindicated for people having a liver disease. The protocol of breast cancer treatment does not take into account this contraindication and the physicians have to substitute tamoxifen by another treatment having the same therapeutic benefit (or a similar therapeutic benefit). For example, they can use anti-aromatases (a drug not contraindicated for people suffering from the liver) instead of tamoxifen or a treatment consisting of the ablation of ovaries (that are organs producing oestrogen).

This example can be formalized as follows. The protocol rules leading to a recommendation of tamoxifen are formalized by $c_{1} \Rightarrow \operatorname{tam}, c_{2} \Rightarrow \operatorname{tam}, \ldots c_{n} \Rightarrow \operatorname{tam}$. This can be expressed by a single rule $c \Rightarrow \operatorname{tam}$, where $c=c_{1} \vee c_{2} \vee \ldots \vee c_{n}$. This rule corresponds to a source case $(\operatorname{srce}, \operatorname{Sol}(\operatorname{srce}))$ with $\operatorname{srce}=c$ and $\operatorname{Sol}(\operatorname{srce})=t a m$. Now, let us consider a woman suffering from breast cancer such that (1) the application of the protocol gives tamoxifen and (2) she suffers from a liver disease. This medical case can be formalized by tgt $=\gamma \wedge$ liver-disease, where $\gamma$ is such that $\gamma \vDash_{\mathrm{DK}} c$ (see below). The domain knowledge is:

$$
\begin{aligned}
\mathrm{DK}= & \gamma \Rightarrow c \wedge \text { liver-disease } \Rightarrow \neg \text { tam } \wedge \text { tam } \Rightarrow \text { anti-oestrogen } \wedge \\
& \text { anti-aromatases } \Rightarrow \text { anti-oestrogen } \wedge \text { ovary-ablation } \Rightarrow \text { anti-oestrogen }
\end{aligned}
$$

liver-disease $\Rightarrow \neg$ tam represents the contraindication of tamoxifen for people suffering from a liver disease. tam $\Rightarrow$ anti-oestrogen (resp., anti-aromatases $\Rightarrow$ anti-oestrogen, ovary-ablation $\Rightarrow$ anti-oestrogen) indicates that if tamoxifen (resp. anti-aromatases, ablation of ovaries) is recommended then an anti-oestrogen treatment is recommended.

The ${ }^{\mathrm{D}}$-conservative adaptation leads to:

$$
\begin{aligned}
\mathrm{TSKCA} & =(\mathrm{DK} \wedge c \wedge \text { tam }){ }_{\mathrm{D}}(\mathrm{DK} \wedge \gamma \wedge \text { liver-disease }) \\
& \equiv_{\mathrm{DK}} \gamma \wedge c \wedge \neg \text { tam } \wedge \text { anti-oestrogen }
\end{aligned}
$$

If the only anti-oestrogen treatments besides tamoxifen are constituted by anti-aromatases and ablation of ovaries then an additional piece of knowledge can be added to DK: anti-oestrogen $\Rightarrow($ tam $\vee$ anti-aromatases $\vee$ ovary-ablation $)$. With this additional knowledge, anti-aromatases $\vee$ ovary-ablation is involved by TSKCA. It can be noticed that this example is very similar to example 1: meat is (in a sense) contraindicated by vegetarians.

Example 3 The large majority of persons suffering from breast cancer are woman (about 99\%). This explains why the protocol of breast cancer treatment has been written for them. When the physicians are confronted to the medical case of a man suffering from this disease, they adapt the protocol. For example, let us consider a man with some 
characteristics $c$, such that, for a woman with these characteristics, the protocol recommends a radical mastectomy (surgery consisting of a breast ablation), a "FEC 100" chemotherapy and an ovary ablation. Both the surgery and the chemotherapy can be applied efficiently to the man, but no ovary ablation (for obvious reasons). The adaptation usually consists in keeping the surgery and the chemotherapy and in substituting the ovary ablation by an anti-oestrogen treatment, such as tamoxifen or anti-aromatases.

The protocol rule used in this example is the source case (srce, Sol(srce)) with srce $=c \wedge$ woman and Sol $($ srce $)=$ radical-mastectomy $\wedge F E C-100 \wedge$ ovary-ablation: radical-mastectomy (resp., FEC-100, ovary-ablation) denotes the persons for which a radical mastectomy (resp., a FEC 100 chemotherapy, an ovary ablation) is recommended. The target problem is tgt $=c \wedge$ man. The domain knowledge is constituted by the domain knowledge of example 2 (denoted hereafter by $\mathrm{DK}_{\mathrm{ex} .2}$ ), the fact that ovary ablation is impossible for men, and the fact that men are not women:

$$
\mathrm{DK}=\mathrm{DK}_{\mathrm{ex} .2} \wedge \text { man } \Rightarrow \neg \text { ovary-ablation } \wedge \neg \text { woman } \vee \neg \text { man }
$$

The result of conservative adaptation, TSKCA, is such that:

$\mathrm{TSKCA} \equiv_{\mathrm{DK}} c \wedge$ man $\wedge$ radical-mastectomy $\wedge F E C-100 \wedge \neg$ ovary-ablation $\wedge$ anti-oestrogen

If the only available anti-oestrogen therapies are tamoxifen, anti-aromatases, and ovary ablation, then DK can be substituted by

$$
\mathrm{DK}^{\prime}=\mathrm{DK} \wedge(\text { anti-oestrogen } \Rightarrow \text { tam } \vee \text { anti-aromatases } \vee \text { ovary-ablation })
$$

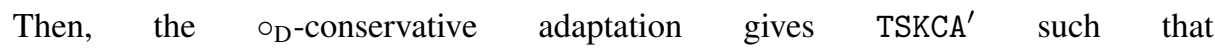
$\mathrm{TSKCA}^{\prime} \equiv \mathrm{TSKCA} \wedge($ tam $\vee$ anti-aromatases $)$.

\section{Discussion}

There have been several proposals in the CBR literature of adaptation approach taxonomies. In [14], conservative adaptation is situated among several such taxonomies. Below, the main part of this work is presented.

Conservative Adaptation and Adaptation by Generalization and Specialization. In [1] is introduced the abstraction and respecialization approach to adaptation that consists in (1) abstracting the solution Sol(srce) of srce into a solution $\mathrm{Sol}(\mathrm{A})$ of an abstract problem A, and (2) specializing Sol(A) in order to solve tgt. According to [15], this adaptation can be better qualified as a generalization/specialization approach (versus an abstraction/refinement approach), but this distinction is not made in [1].

The examples of conservative adaptations presented in this paper may be seen as the application of some generalization and specialization adaptations. For instance, in example 3, Sol(srce) is generalized by substituting ovary-ablation by anti-oestrogen and then, whenever it is known that the only available anti-oestrogen treatments besides ovary ablation are tamoxifen and anti-aromatases, anti-oestrogen is specialized into tam $\vee$ anti-aromatases. 
This behavior of $\mathrm{O}_{\mathrm{D}}$-conservative adaptation can be understood thanks to a definition of distance-based revision operators $\circ_{\text {dist }}\left(\right.$ such as $\circ_{D}$ ), equivalent to the one given in section 3.3 and inspired from [7]. This definition is as follows. First, for any real number $\delta \geq 0$, let $G^{\delta}$ be a function that maps a propositional formula $\psi$ based on a set of variables $\mathcal{V}$ to another formula $G^{\delta}(\psi)$ on $\mathcal{V}$, such that

$$
\operatorname{Mod}\left(G^{\delta}(\psi)\right)=\{\mathcal{I} \mid \mathcal{I}: \text { interpretation on } \mathcal{V} \text { and } \operatorname{dist}(\operatorname{Mod}(\psi), \mathcal{I}) \leq \delta\}
$$

$G^{\delta}$ realizes a generalization: $\psi \vDash G^{\delta}(\psi)$ for any $\psi$ and any $\delta$. Moreover $G^{0}(\psi) \equiv \psi$. Finally, if $0 \leq \delta \leq \varepsilon$, then $G^{\delta}(\psi) \vDash G^{\varepsilon}(\psi)$. For $\psi$ and $\mu$, two satisfiable formulas on $\mathcal{V}$, let $\Delta$ be the least value $\delta$ such that $G^{\delta}(\psi) \wedge \mu$ is satisfiable. ${ }^{1}$ Then, $\psi \circ_{\text {dist }} \mu$ can be defined by $\psi \circ_{\text {dist }} \mu=G^{\Delta}(\psi) \wedge \mu$. If either $\psi$ or $\mu$ is unsatisfiable, then $\psi \circ_{\text {dist }} \mu \equiv \mu$. It can be proven easily that this definition of $\circ_{\text {dist }}$ is equivalent to the one of section 3.3 (as soon as syntax is considered to be irrelevant). Thus $\psi \circ_{\text {dist }} \mu$ can be interpreted as follows: it is obtained by generalizing $\psi$ in a minimal way (according to the scale $\left.\left(\left\{G^{\delta}(\psi)\right\}_{\delta}, \vDash\right)\right)$ in order to be consistent with $\mu$, and then, it is specialized by a conjunction with $\mu$.

Conservative Adaptation and Problem Decomposition. In [16], adaptation is considered within three taxonomies. One of them is the taxonomy of the adaptation operators used in adaptation procedures. Let us consider two of these operators: (1) subgoaling operators and (2) goal interaction operators. (1) A subgoaling operator aims at decomposing the adaptation task into subtasks while (2) a goal interaction operator handles interactions between solution parts: it detects and repairs bad interactions. It may be considered that conservative adaptation performs a combination of operations of types (1) and (2). The specification of a target problem -the formula tgt- can be viewed as a goal specification (the goal is to find a solution consistent with tgt). If tgt $\equiv \operatorname{tgt}_{1} \wedge \operatorname{tgt}_{2}$ then $\operatorname{tgt}_{1}$ and $\operatorname{tgt}_{2}$ are two subgoals of the target problem. Conservative adaptation provides a solution that is consistent with both subgoals. Therefore, this approach to adaptation considers possibly interacting subgoals as a combined use of operators of types (1) and (2) would do. However, if the revision operator is considered as a black box, then the distinction between (1) and (2) operators is not visible.

Conservative Adaptation and Copy-Modify-Test Approach to Adaptation. In [17], a general model of adaptation in CBR is presented in a task formalism: starting from the analysis of several CBR systems implementing an adaptation process, they propose a hierarchical decomposition of adaptation in tasks and subtasks. The idea is that many (if not all) transformational adaptation procedures implemented in CBR systems may

\footnotetext{
${ }^{1}$ In fact, $\Delta=\operatorname{dist}(\operatorname{Mod}(\psi), \operatorname{Mod}(\mu))$ realizes this: $(a) G^{\Delta}(\psi) \wedge \mu$ is satisfiable and $(b)$ if $\delta<\Delta$ then $G^{\delta}(\psi) \wedge \mu$ is unsatisfiable. (a) can be proven as follows. Let $\mathcal{J} \in \operatorname{Mod}(\mu)$ such that $\Delta=\operatorname{dist}(\operatorname{Mod}(\psi), \mathcal{J})$ (this makes sense since $\operatorname{Mod}(\psi) \neq \emptyset)$. Thus, $\mathcal{J}$ also belongs to $\operatorname{Mod}\left(G^{\Delta}(\psi)\right)$ and so $\mathcal{J} \in \operatorname{Mod}\left(G^{\Delta}(\psi)\right) \cap \operatorname{Mod}(\mu)=\operatorname{Mod}\left(G^{\Delta}(\psi) \wedge \mu\right)$, which proves $(a)$. (b) can be proven by contradiction, assuming that there is some $\delta<\Delta$ such that $G^{\delta}(\psi) \wedge \mu$ is satisfiable. If so, let $\mathcal{J} \in \operatorname{Mod}\left(G^{\delta}(\psi) \wedge \mu\right)$, thus $\mathcal{J} \in \operatorname{Mod}\left(G^{\delta}(\psi)\right)$ and $\mathcal{J} \in \operatorname{Mod}(\mu)$. Therefore $\Delta=\operatorname{dist}(\operatorname{Mod}(\psi), \operatorname{Mod}(\mu)) \leq \delta$, which is in contradiction with the assumption $\delta<\Delta$. Thus, (b) is also proven.
} 
be modelled according to this scheme, considering a subset of these tasks. Conservative adaptation may be seen as a way of instantiating the following subset of tasks:

- Copy solution (that is similar to null adaptation);

- Select and modify discrepancies (by removing, substituting, and/or adding some pieces of information using the domain knowledge);

- Test consistency.

In fact, in conservative adaptation, it is the revision operator that processes all these tasks: it performs a minimal change that can be seen as a sequence of copy, modification, and test tasks. Moreover, it uses the domain knowledge in order to choose the features to be modified in order to reach consistency.

Therefore, conservative adaptation may also be seen as an instanciation of the reuse and revise steps of the Aamodt and Plaza's cycle [18]: reuse is performed by a simple copy and revise by a revision operator. It can be noticed that, to our knowledge, the revise step of the CBR cycle has not been related to the AGM theory of revision: we have found only one paper on CBR using revision techniques [19], but not for the purpose of the reasoning process itself, but for the maintenance of the case base and of a rule base when there are some evolutions in time (according to [20], this is more an update of a knowledge base than a revision of it).

\section{Conclusion and Future Work}

In case-based reasoning, adaptation is often considered as a difficult task, in comparison to retrieval that is supposed to be simpler to design and to implement. This paper presents an approach to adaptation, called conservative adaptation, that is based on the theory of revision: it consists in keeping as much as possible from the source case while being consistent with the target problem and the domain knowledge. Conservative adaptation is defined, formalized in the framework of propositional logic, and this formalism can be extended to other knowledge representation formalisms. Moreover, it is shown through examples that conservative adaptation covers some of the adaptations performed by experts in oncology. This approach to adaptation can be used for knowledge-intensive approaches to CBR, since it requires some domain knowledge. A noticeable feature of conservative adaptation is that the adaptation knowledge is part of the domain knowledge: it is not constituted by, e.g., a set of adaptation rules. This paper has also shown that the AGM theory of revision and the huge amount of research based on this theory may be of interest for adaptation in CBR: a revision operator should be considered as a tool for designing a conservative adaptation procedure.

Section 6 shows that conservative adaptation shares some common features with general approaches to adaptation defined in the CBR literature, in particular handling the problems of consistency, extending null adaptation (also called copy of the source solution), and, at least for $\circ_{\text {dist }}$-conservative adaptation, being equivalent to an adaptation by generalization and specialization.

Several theoretical issues about conservative adaptation have been addressed in the research report [14], that deserve further investigations. Some of them are listed hereafter. One of them is the design of a retrieval procedure suited to conservative adaptation. It is based on the assumption that a conservative adaptation process is better than 
another one if the former requires less change thant the latter. This leads to prefer the source case $\left(\operatorname{srce}^{1}, \operatorname{Sol}\left(\operatorname{srce}^{1}\right)\right)$ to the source case $\left(\operatorname{srce}^{2}, \operatorname{Sol}\left(\operatorname{srce}^{2}\right)\right)$ if the former requires less change than the latter, which amounts to $\Delta^{1}<\Delta^{2}$, with

$$
\Delta^{i}=\operatorname{dist}\left(\operatorname{Mod}\left(\mathrm{DK} \wedge \operatorname{srce}^{i} \wedge \operatorname{Sol}\left(\operatorname{srce}^{i}\right)\right), \operatorname{Mod}(\mathrm{DK} \wedge \operatorname{tgt})\right) \quad(i \in\{1,2\})
$$

However, on the one hand, this preference criterion may be insufficient to distinguish two source cases and, on the other hand, its naive implementation is intractable.

The knowledge required for conservative adaptation is the domain knowledge DK of the CBR system under consideration: DK is useful to point out the features of the source case that need to be adapted to the context of the target problem. Thus, with insufficient domain knowledge, conservative adaptation may provide an unsatisfying solution to the target problem: this solution contradicts the expert knowledge but does not contradict DK. In other words, the failed result of conservative adaptation is due to the gap between DK and the expert knowledge (a gap that cannot be completely filled in practice, due to the qualification problem mentioned in section 5.1). Thus, from an analysis of the failure, some new domain knowledge can be acquired and added to the current DK. Therefore, a CBR system may learn new domain knowledge from the explanations that follow failed conservative adaptation, which involves an improvement of its competence. The paper [21] proposes an approach to address this issue together with the description of a prototype that implements this approach.

Conservative adaptation covers only a part of the adaptations actually performed by experts. Some other adaptations could be covered thanks to extensions of conservative adaptation, as shown in the research report [14]. In particular, an approach to adaptation

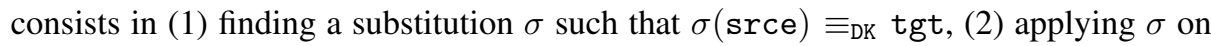
$\mathrm{Sol}$ (srce) to provide a first solution of $\mathrm{Sol}_{1}(\mathrm{tgt})$, and (3) repairing $\mathrm{Sol}_{1}$ (tgt) to make it consistent with the domain knowledge. The step (3) can be performed by a revision operator. For instance, in [14], the well-known example of "beef and broccoli adaptation" of the CHEF system [22] is re-described using the revision operator ${ }_{\mathrm{D}}$. This also shows, more generally, that revision operators can be used in various ways as tools for designing and implementing adaptation processes. The study of such extensions is another research direction.

Another future work is the combination of several source cases $\left(\operatorname{srce}^{1}, \operatorname{Sol}\left(\operatorname{srce}^{1}\right)\right), \ldots\left(\operatorname{srce}^{n}, \operatorname{Sol}\left(\operatorname{srce}^{n}\right)\right)$ to solve a sole target problem tgt. It is planned to study this issue thanks to the notion of merging of propositional knowledge bases [23]: given a multiset $\left\{\psi_{1}, \ldots \psi_{n}\right\}$ of knowledge bases to be merged and a consistent knowledge base $\mu$ (the "integrity constraint"), a merging operator builds a knowledge base $\triangle_{\mu}\left(\left\{\psi_{1}, \ldots \psi_{n}\right\}\right)$ that is consistent with $\mu$ and keeps "as much as possible" information from the $\psi_{i}$ 's. This extends the notion of revision: $\circ$ defined by $\psi \circ \mu=\triangle_{\mu}(\{\psi\})$ is a revision operator. In the same way, an approach to case combination that extends conservative adaptation is to compute $\triangle_{\mu}\left(\left\{\psi_{1}, \ldots \psi_{n}\right\}\right)$ with $\psi_{i}=\mathrm{DK} \wedge \operatorname{srce}^{i} \wedge \mathrm{Sol}\left(\operatorname{srce}^{i}\right)(i \in\{1,2, \ldots n\})$ and $\mu=\mathrm{DK} \wedge$ tgt. The relevance of this approach for practical problems of case combination in CBR remains to be studied.

From a practical viewpoint, future work will be the development and the use of a conservative adaptation tool to be integrated within the KASIMIR system. A first tool implementing the Dalal's revision operator has been implemented, but it can be optimized. As an example, the most complex computation of a revision presented in [14] 
is based on 16 propositional variables and requires about 25 seconds on a current PC. Another practical issue is the integration of conservative adaptation in the KASIMIR system, which raises two problems. The first one is that both the cases and the domain knowledge of KASIMIR are represented in a formalism equivalent to the description logic $\mathcal{S H O I N}$ (D). Therefore, either adaptation problems expressed in $\mathcal{S H O I N}$ (D) are translated in propositional logic and solved in this formalism, or a revision operator has to be implemented for a description logic compatible with KASIMIR (this second solution requires a formalization of conservative adaptation in description logics; a first proposal of such a formalization is given in [14]). When this integration issue is addressed, a comprehensive evaluation of the scope of conservative adaptation can be carried out. The second problem of integration is linked with the already existing adaptation module of KASIMIR [12], that is based on adaptation rules (roughly speaking). How conservative adaptation and this rule-based adaptation module can be integrated together in order to provide a unique adaptation module enabling complex adaptation processes (each of them being composed of a conservative adaptation and some rule-based adaptations)? This question should be addressed thanks to earlier work on adaptation composition and decomposition [24].

\section{Acknowledgements}

The author would like to thank greatly Pierre Marquis who, some years ago, has taught him the basis of the revision theory, has, more recently, suggested some interesting references about this theory, has made some interesting remarks on an earlier version of this paper, and has suggested him to use merging operators for case combination. $\mathrm{He}$ also wants to thank his beloved daughter who is, for some strange reason, at the origin of this study. Finally, he thanks the reviewers for their interesting comments.

\section{References}

1. C. K. Riesbeck and R. C. Schank. Inside Case-Based Reasoning. Lawrence Erlbaum Associates, Inc., Hillsdale, New Jersey, 1989.

2. A. Aamodt. Knowledge-Intensive Case-Based Reasoning and Sustained Learning. In L. C. Aiello, editor, Proceedings of the 9th European Conference on Artificial Intelligence (ECAI'90), August 1990.

3. D. Dubois, F. Esteva, P. Garcia, L. Godo, R. L. de Màntaras, and H. Prade. Fuzzy set modelling in case-based reasoning. Int. J. of Intelligent Systems, 13:345-373, 1998.

4. M. d'Aquin, J. Lieber, and A. Napoli. Adaptation Knowledge Acquisition: a Case Study for Case-Based Decision Support in Oncology. Computational Intelligence (an International Journal), 22(3/4):161-176, 2006.

5. C. E. Alchourrón, P. Gärdenfors, and D. Makinson. On the Logic of Theory Change: partial meet functions for contraction and revision. Journal of Symbolic Logic, 50:510-530, 1985.

6. H. Katsuno and A. Mendelzon. Propositional knowledge base revision and minimal change. Artificial Intelligence, 52(3):263-294, 1991.

7. M. Dalal. Investigations into a theory of knowledge base revision: Preliminary report. In AAAI, pages 475-479, 1988. 
8. J. McCarthy. Epistemological Problems of Artificial Intelligence. In Proceedings of the 5th International Joint Conference on Artificial Intelligence (IJCAI'77), Cambridge (Massachussetts), pages 1038-1044, 1977.

9. K. Maximini, R. Maximini, and R. Bergmann. An investigation of generalized cases. In K. D. Ashley and D. Bridge, editors, Proceedings of the 5th International Conference on Case Base Reasoning (ICCBR'03), volume 2689 of LNAI, pages 261-275, Trondheim, Norway, June 2003. Springer.

10. D. Fensel, J. Hendler, H. Lieberman, and W. Wahlster, editors. Spinning the Semantic Web. The MIT Press, Cambridge, Massachusetts, 2003.

11. S. Staab and R. Studer, editors. Handbook on Ontologies. Springer, Berlin, 2004.

12. M. d'Aquin, J. Lieber, and A. Napoli. Case-Based Reasoning within Semantic Web Technologies. In Twelfth International Conference on Artificial Intelligence: Methodology, Systems, Applications (AIMSA-06), pages 190-200, 2006.

13. M. d'Aquin, F. Badra, S. Lafrogne, J. Lieber, A. Napoli, and L. Szathmary. Case Base Mining for Adaptation Knowledge Acquisition. In M. M. Veloso, editor, Proceedings of the 20th International Joint Conference on Artificial Intelligence (IJCAI'07), pages 750-755. Morgan Kaufmann, Inc., 2007.

14. J. Lieber. A Definition and a Formalization of Conservative Adaptation for KnowledgeIntensive Case-Based Reasoning - Application to Decision Support in Oncology (A Preliminary Report). Research report, LORIA, 2006.

15. R. Bergmann. Learning Plan Abstractions. In H. J. Ohlbach, editor, GWAI-92, $16^{\text {th }}$ German Workshop on Artificial Intelligence, Lecture Notes in Artificial Intelligence 671, pages 187198. Springer Verlag, Berlin, 1992.

16. K. Hanney, M. T. Keane, B. Smyth, and P. Cunningham. Systems, Tasks and Adaptation Knowledge: Revealing Some Revealing Dependencies. In M. Veloso and A. Aamodt, editors, Case-Based Reasoning Research and Development - First International Conference, ICCBR'95, Sesimbra, Portugal, LNAI 1010, pages 461-470. Springer Verlag, Berlin, 1995.

17. B. Fuchs and A. Mille. A Knowledge-Level Task Model of Adaptation in Case-Based Reasoning. In K.-D. Althoff, R. Bergmann, and L. K. Branting, editors, Case-Based Reasoning Research and Development - Third International Conference on Case-Based Reasoning (ICCBR-99), Lecture Notes in Artificial Intelligence 1650, pages 118-131. Springer, 1999.

18. A. Aamodt and E. Plaza. Case-based Reasoning: Foundational Issues, Methodological Variations, and System Approaches. AI Communications, 7(1):39-59, 1994.

19. R. Pavón Rial, R. Laza Fidalgo, A. Gómez Rodriguez, and J. M. Corchado Rodriguez. Improving the Revision Stage of a CBR System with Belief Revision Techniques. Computing and information systems journal, 8(2):40-45, 2001.

20. H. Katsuno and A. Mendelzon. On the Difference Between Updating a Knowledge Base and Revising It. In James F. Allen, Richard Fikes, and Erik Sandewall, editors, KR'91: Principles of Knowledge Representation and Reasoning, pages 387-394. Morgan Kaufmann, San Mateo, California, 1991.

21. A. Cordier, B. Fuchs, J. Lieber, and A. Mille. Failure Analysis for Domain Knowledge Acquisition in a Knowledge-Intensive CBR System. In this volume.

22. K. J. Hammond. Case-Based Planning: A Framework for Planning from Experience. Cognitive Science, 14(3):385-443, 1990.

23. S. Konieczny, J. Lang, and P. Marquis. $\mathrm{DA}^{2}$ merging operators. Artificial Intelligence, 157(1-2):49-79, 2004.

24. J. Lieber. Reformulations and Adaptation Decomposition. In J. Lieber, E. Melis, A. Mille, and A. Napoli, editors, Formalisation of Adaptation in Case-Based Reasoning. Third International Conference on Case-Based Reasoning Workshop, ICCBR-99 Workshop number 3, S. Schmitt and I. Vollrath (volume editor), LSA, University of Kaiserslautern, 1999. 